\title{
Efficacy evaluation and mechanical study of short- and long-term antithrombotic therapy for Kawasaki disease
}

\author{
Jingxia Hao, Hua Wang, Jingshi Chen, Bo Li, Huimin Zhang, Yingqian Zhang \\ Department of Cardiology, Hebei Children's Hospital, Shijiazhuang, China \\ Contributions: (I) Conception and design: Y Zhang (II) Administrative support: J Hao; (III) Provision of study materials or patients: H Wang, J Chen; \\ (IV) Collection and assembly of data: J Hao, B Li; (V) Data analysis and interpretation: J Hao, H Zhang; (VI) Manuscript writing: All authors; \\ (VII) Final approval of manuscript: All authors. \\ Correspondence to: Yingqian Zhang. Department of Cardiology, Hebei Children's Hospital, 133 Jianhua Street, Shijiazhuang 050031, China. \\ Email: 251654155@qq.com.
}

Background: Antithrombotic therapy was commonly applied in treating Kawasaki disease (KD) children; however, the effects and mechanisms of different plans were not fully elucidated. In this study, we aimed to evaluate different antithrombotic drugs.

Methods: Eighty-two children diagnosed with KD in Hebei Children's Hospital from January 2017 to January 2020 were recruited. For cohort study, KD children were divided into a series of groups according to whether they were complicated with coronary artery lesions (CAL), drug therapy plan, and the presence of liver damage. The thromboelastogram (TEG) indexes [clotting time (R), clot formation time (K), clot formation angle $(\alpha)$, maximum amplitude of the clot (MA), arachidonic acid (AA), and adenosine diphosphate (ADP)] were employed to evaluate the relationship between disease state and drug treatment efficacy. Meanwhile, children were divided into different therapy groups according to their degree of CAL, the treatment efficiency was evaluated by TEG indexes, and the bleeding ratio was recorded. In addition, the warfarin metabolic gene was detected to explain the changes of coagulation parameters in children treated with warfarin.

Results: The $R$ value and coagulation index $(\mathrm{CoI})$ were significantly lower $(\mathrm{P}<0.05)$ and MA value was significantly higher $(\mathrm{P}<0.05)$ in $\mathrm{CAL}$ group than those in non-coronary artery lesion $(\mathrm{NCAL})$ group. There were significant individual differences in platelet inhibition between aspirin and dipyridamole groups. The AA $\%$ in aspirin group was $64 \%$ [95\% confidence interval (CI): $49 \%$ to $74.3 \%$ ] and the ADP\% was $28.5 \%$ (95\% CI: $26.2 \%$ to $37.2 \%$ ) in dipyridamole group, and were significantly between groups. Warfarin and aspirin had a synergistic effect in anticoagulation. Warfarin metabolic gene detection was shown to be helpful for adjusting warfarin treatment dose, and shortening the initial attainment time of standard international normalized ratio (INR) index. The $\mathrm{R}$ value was significantly higher $(\mathrm{P}<0.05)$ in the liver injury group than that in the control group.

Conclusions: KD Children complicated with CAL presented a hypercoagulable state, and the CAL was predictable with the $\mathrm{R}$ value. Warfarin metabolic gene detection and TEG can effectively evaluate and guide short- and long-term antithrombotic therapy in $\mathrm{KD}$ children.

Keywords: Kawasaki disease (KD); coronary artery lesion (CAL); coagulation function; thrombelastography; gene polymorphism detection.

Submitted Jun 02, 2021. Accepted for publication Jul 19, 2021.

doi: 10.21037/tp-21-299

View this article at: https://dx.doi.org/10.21037/tp-21-299 


\section{Introduction}

Mucocutaneous lymph node syndrome (MCLS), also called Kawasaki disease (KD), is an acute self-limited vasculitis of unknown etiology, which mainly occurs in children under 5 years old (1). The main clinical manifestations of $\mathrm{KD}$ are as follows: persistent fever for over 5 days, bulbar conjunctiva hyperemia, neck lymphadenopathy, raspberry tongue, skin rash, and dry, cracked lips. Additionally, in the acute phase, children with KD manifest non-pitting swollen of the hands and feet, and the fingers, toes, and perianal skin begin desquamate in the recovery period (2). Research has indicated that the long-term prognosis of $\mathrm{KD}$ depends on the situation of the coronary artery, and children with coronary artery lesions (CAL) may even experience myocardial infarction (3). Previous studies have shown that the occurrence of CAL is closely related to the coagulation state. In this study, we analyzed the correlation of coagulation function with the occurrence of CAL in children with acute stage KD, evaluated the therapeutic effects of different antithrombotic treatments with thromboelastogram (TEG) index, and explored the related mechanisms. Meanwhile, we further analyzed the effect of warfarin metabolic-related gene polymorphism on the dose selection in children with $\mathrm{KD}$ complicated with coronary artery disease.

We present the following article in accordance with the STROBE reporting checklist (available at https://dx.doi. org/10.21037/tp-21-299).

\section{Methods}

\section{Study design and participants}

This study was approved by the Ethics Committee of Hebei Children's Hospital (2017005), all participants were well informed, and written informed consent was provided by the guardians of participants. A total of 82 children who were diagnosed with $\mathrm{KD}$ and received treatment in Hebei Children's Hospital from January 2017 to January 2020 were recruited to this cohort study. In the cohort study, the bleeding ratio of participants was followed up for 3 months. The diagnosis of $\mathrm{KD}$ was made according to the 2017 edition of Diagnosis, Treatment, and Long-term Management of Kawasaki Disease: A Scientific Statement for Health Professionals from the American Heart Association (1). Heart ultrasound was used to examine the coronary artery, and the internal diameter ( $\mathrm{Z}$ value) of any segment of the coronary artery $\geq 2.0$ was diagnosed as $\mathrm{KD}$ complicated with CAL, which determined the degree of
KD. The children were divided into the corresponding groups according to the grouping standards and treatment received. The liver functions, blood routine, and TEG indexes were examined and analyzed. The study was conducted in accordance with the Declaration of Helsinki (as revised in 2013).

\section{Inclusion and exclusion criteria}

The inclusion criteria were as follows: (I) children were diagnosed with $\mathrm{KD}$ exactly according to the standard; (II) consent to participate in this study.

The exclusion criteria were as follows: (I) children complicated with coagulopathy, severe infection, shock, and macrophage activation; (II) children who interrupted anticoagulation or changed antiplatelet therapy plan during the course of treatment.

\section{Grouping and observation indexes}

\section{CAL and non-CAL groups}

The KD children were divided into the CAL group and non-CAL (NCAL) group according to whether they were complicated with CAL when in-hospital. The TEG indexes were compared between 2 groups before they were administered with antithrombotic therapy, exploring the relationship between coagulation function and KD complicated with CAL.

\section{Liver damage and non-liver damage groups}

According to their liver functions before treatment, KD children were allocated to a liver damage group and nonliver damage group, and the TEG indexes were compared between 2 groups before they received treatment.

\section{Aspirin, dipyridamole, and aspirin combined with warfarin groups}

According to the degree of CAL and the condition of disease, different antithrombotic therapy plans were determined, which included aspirin treatment (AA), dipyridamole treatment (DIP), and aspirin combined with warfarin treatment (AW). Arachidonic acid (AA) $\%>50 \%$ and adenosine diphosphate $(\mathrm{ADP}) \%>30 \%$ were defined as effective, the TEG indexes in different groups were compared, and the effects of antithrombotic therapy were evaluated.

\section{Warfarin individual treatment and routine treatment}

The KD children that were determined to receive warfarin 
therapy were further randomly divided into a warfarin individual treatment group and warfarin routine treatment group. The warfarin metabolic gene of the children in individual treatment groups was further detected. The warfarin effective dose, steady drug concentration dose, and effective time were compared between the 2 groups, and the role of gene polymorphism in antithrombotic therapy was analyzed.

\section{Treatment strategy}

All children diagnosed with KD received a single dose of human immunoglobulin $(2 \mathrm{~g} / \mathrm{kg})$ treatment, as well as oral intake of AA or DIP. The initial dose of AA was $30-50 \mathrm{mg} /(\mathrm{kg} \cdot \mathrm{d})$, which was reduced to $3-5 \mathrm{mg} /(\mathrm{kg} \cdot \mathrm{d})$ after the fever had been gone for 48-72 h, and the dose of DIP was $3-5 \mathrm{mg} /(\mathrm{kg} \cdot \mathrm{d})$. The grouping standards of drug treatment were as follows: routine aspirin treatment group (AA), children complicated with aspirin allergy, severe liver dysfunction, influenza virus, or varicella virus infection were allocated to the DIP treatment group. The KD children complicated with IV degree CAL were administered low molecular weight heparin (LMWH) in the acute phase. If the lesion stopped progressing, and the condition of disease was controlled, LMWH was changed to warfarin treatment, and this category of children were divided into the AW group. Routine warfarin treatment was applied in the negative warfarin metabolic gene detection group, the initial dose was 0.05 $\mathrm{mg} /(\mathrm{kg} \cdot \mathrm{d})$, and the dose was adjusted according to the international normalized ratio (INR), to maintain an INR value between 1.5 and 2.5. Warfarin individual treatment was applied in the group that tested positive for the warfarin metabolic gene polymorphism, and the dose was determined according to the gene detection results.

\section{Examination methods and indexes}

All blood samples were collected from KD children via venipuncture before the immunoglobulin was injected or in the early morning on an empty stomach before high dose aspirin was decreased to minimal dose for antiplatelet treatment. The children's liver functions, blood routine were monitored twice a week, and TEG once a week. The TEG Analyser-5000 (Haemoscope, Niles, IL, USA) was used to examine the TEG indexes including clotting time ( $\mathrm{R}$ value), clot formation time ( $\mathrm{K}$ value), clot formation ratio ( $\alpha$ angle), maximum amplitude of the clot (MA), and coagulation index (CoI). The TEG indexes were compared among all the groups with statistical analysis.

\section{Statistical analysis}

The SPSS 17.0 software (IBM Corp., Chicago, IL, USA) was used for statistical analysis among groups. Normally distributed data were presented as mean \pm standard deviation (SD), and the non-normally distributed quantitative data were presented using interquartile range ( $25 \%$ to $75 \%$ ). A homogeneity test of variance was carried out before analysis of variance (ANOVA) was used to analyze the differences among groups, and a nonparametric test (rank-sum test) was used for the heterogeneous variance data. The differences between subgroups were analyzed with Scheffé test; $\mathrm{P}<0.05$ was considered statistical significant.

\section{Results}

\section{Comparison of coagulation related indexes between CAL group and NCAL group}

The alanine aminotransferase (ALT), aspartate transaminase (AST), platelet count test (Plt), C-reactive protein (CRP), and procalcitonin (PCT) of $82 \mathrm{KD}$ children were compared between the CAL group and NCAL group. The results showed that there were no differences in all indexes between 2 groups, and none of the children had bleeding during the follow up period. Table 1 shows the results of TEG indexes ( $\mathrm{R}$ value, $\mathrm{K}$ value, $\alpha$ angle, MA, and $\mathrm{CoI}$ ) compared between the CAL group and NCAL group: the R value, MA, and the CoI showed significant differences $(\mathrm{P}<0.05)$, while other indexes showed no difference between 2 groups.

\section{Comparison of coagulation related indexes between liver damage group and non-liver damage group}

The TEG indexes were compared between the liver damage group and non-liver damage group and are presented in Table 2. The results showed that $\mathrm{R}$ value was significantly different $(\mathrm{P}<0.05)$ between 2 groups, and no differences were detected between the remaining indexes.

\section{Comparison of antiplatelet effects among $A A$ group, DIP group, and $A W$ group}

The data of platelet inhibition ratio after KD children underwent antiplatelet treatment was normally distributed. 
Table 1 Comparison of TEG indexes between CAL group and NCAL group

\begin{tabular}{lcccccc}
\hline Groups & Numbers & R value & K value & $\alpha$ angle & MA & Col \\
\hline CAL & 30 & $3.58 \pm 1.08$ & $1.69 \pm 0.50$ & $65.78 \pm 9.04$ & $71.46 \pm 9.31$ & $1.75 \pm 1.42$ \\
NCAL & 52 & $4.32 \pm 1.26$ & $1.73 \pm 0.51$ & $67.61 \pm 5.43$ & $58.57 \pm 9.69$ & $2.39 \pm 1.31$ \\
$t$ value & & -2.68 & -0.23 & -1.1 & 5.89 & 2.066 \\
P value & & 0.01 & 0.75 & 0.25 & 0.00 & 0.042 \\
\hline
\end{tabular}

TEG, thromboelastogram; CAL, coronary artery lesion; NCAL, non-coronary artery lesion; $\mathrm{R}$ value, clotting time; $\mathrm{K}$ value, clot formation time; $\alpha$ angle, clot formation ratio; MA, maximum amplitude of the clot; Col, coagulation index.

Table 2 Comparison of TEG indexes between liver injury group and non-liver injury group

\begin{tabular}{lcccccc}
\hline Groups & Numbers & R value & K value & $\alpha$ angle & MA & Col \\
\hline liver injury & 35 & $5.65 \pm 1.91^{*}$ & $1.63 \pm 0.53$ & $68.33 \pm 5.70$ & $63.36 \pm 10.26$ & $2.28 \pm 1.43$ \\
non-liver injury & 47 & $4.14 \pm 1.15$ & $1.78 \pm 0.48$ & $65.91 \pm 7.70$ & $61.95 \pm 10.98$ & $2.06 \pm 1.34$ \\
$t$ value & & -4.47 & 1.39 & -1.57 & -0.59 & -0.70 \\
P value & & 0.00 & 0.17 & 0.12 & 0.55 & 0.48 \\
\hline
\end{tabular}

TEG, thromboelastogram; CAL, coronary artery lesion; NCAL, non- coronary artery lesion; $\mathrm{R}$ value, clotting time; $\mathrm{K}$ value, clot formation time; $\alpha$ angle, clot formation ratio; MA, maximum amplitude of the clot; Col, coagulation index; * means $\mathrm{P}<0.05$

Table 3 Comparison of treatment efficiency among three different treatment plans

\begin{tabular}{lcccc}
\hline Therapy plan & Number of effective & Number of ineffective & $\chi^{2}$ & P value \\
\hline AA & 37 & 12 & 4.7 & 0.04 \\
DIP & 8 & 9 & 4.8 & 0.03 \\
AW & 16 & 0 & \\
\hline
\end{tabular}

AA, aspirin group; DIP, dipyridamole group; AW, aspirin combined with warfarin; all the groups were compared to AA group.

Comparison of the platelet inhibition ratio between the AA group and DIP group showed that the AA\% was 64\% (49\%, $74.3 \%)$ in the AA group and $72.5 \%(69 \%, 90.9 \%)$ in the $\mathrm{AW}$ group; the $\mathrm{ADP} \%$ in the DIP group was $28.5 \%$ (26.2\%, $37.2 \%)$. The comparison of the effective efficiency between the AA group and DIP group showed that the effective efficiency was $75.5 \%$ in the AA group, $47 \%$ in DIP group, and $100 \%$ in $\mathrm{AW}$ group; there were statistical differences between the AA group and DIP group $(\mathrm{P}<0.05)$, as well as the AA group and AW group $(\mathrm{P}<0.05)$, and the results are presented in Table 3.

\section{Comparison of the time of initial attainment of standard INR and dosage in the warfarin routine treatment group and individual treatment group}

Table 4 shows the results of the time to first achieve the standard INR value, the initial dosage, and the dose of warfarin when INR reached standard in the routine treatment group and individual treatment group. The INR value first reaching standard time was $10.50 \pm 2.20$ days in the warfarin individual treatment group, and $15.75 \pm 2.92$ days in the routine treatment group, these times were significantly different. Meanwhile, the initial dosage and the steady phase dosage showed no difference between 2 groups.

\section{Discussion}

The illness KD is a self-limited systemic vasculitis, which has exceeded acute rheumatic fever as the leading cause of acquired heart disease in children in most countries (4). In children with KD, the blood is hypercoagulable in the acute phase, complicated with inflammationmediated endothelial dysfunction, abnormal platelet 
Table 4 Comparison of the efficiency and warfarin dose between routine treatment group and individual treatment group

\begin{tabular}{lccc}
\hline Groups & First attainment of standard time (days) & Initial dose of warfarin $(\mathrm{mg} / \mathrm{kg})$ & Dose when INR reach standard $(\mathrm{mg} / \mathrm{kg})$ \\
\hline Individual therapy & $10.50 \pm 2.20$ & $0.06 \pm 0.01$ & $0.09 \pm 0.02$ \\
Routine therapy & $15.75 \pm 2.92^{*}$ & $0.05 \pm 0.01$ & $0.11 \pm 0.02$ \\
$t$ value & -4.063 & 1.63 & -0.79 \\
$\mathrm{P}$ value & 0.001 & 0.13 & 0.45 \\
\hline
\end{tabular}

* means $\mathrm{P}<0.05$.

activation, abnormal fibrinolysis, and hemodynamic changes (5-8). All these mechanisms can induce CAL, resulting in coronary artery dilation or stenosis and forming thrombosis, further contributing to myocardial infarction-induced ischemic cardiomyopathy, or even sudden death (9). Recently, a accumulating studies have shown the correlation of vascular inflammation with blood hypercoagulability in $\mathrm{KD}$, which ultimately leads to CAL $(9,10)$. Hartert invented the method of TEG in 1948 to evaluate the whole process of coagulation and fibrinolysis according to the change of blood clot intensity. Compared with traditional coagulation function tests, TEG can reflect the whole blood's overall coagulation and fibrinolysis ability, including platelet function via the results and TEG curve, and it can also detect the platelet inhibition ratio to evaluate the drugs' antiplatelet effects via TEG-platelet mapping (TEG-PM). Hobson et al. found that (11) a higher MA is associated with increased thrombosis risk, and the correspondence is of high sensitivity and specificity. In this study, we used TEG to examine the coagulation function in children with KD, and the results showed that the $\mathrm{R}$ value and MA were significantly higher in the CAL group than NCAL group, indicating that CAL is closely associated with coagulation dysfunction.

The liver is the main organ for the synthesis and metabolism of coagulation factors (12). When the liver was dysfunctional, the synthesis of coagulation factors and heparinase was decreased, the ability to eliminate tissue thrombin and activated fibrinolytic factors was also impaired, and the absorption of vitamin $\mathrm{K}$ was also decreased, thus eventually contributed to abnormal clotting (4). The pathological basis of $\mathrm{KD}$ is systemic vasculitis, and the vascular abundance of the liver makes it the most easily affected organ (13). Some other studies have indicated that KD-induced liver damage may relate to hepatic vasculitis and damage of the biliary system $(14,15)$. In this study, we found that the $\mathrm{R}$ value of TEG was significantly higher in the liver damage group, while the MA, K value, $\alpha$ angle, and $\mathrm{CoI}$ showed no differences, suggesting that $\mathrm{KD}$ may induce liver injury, and liver damage further disrupted coagulation functions, thus exacerbating the disease condition.

Antithrombotic therapy is an important step in the treatment of KD children, while few studies concerning the efficacy and risk of antithrombotic therapy have been reported (4). The drug AA is widely used in $\mathrm{KD}$ treatment for its anti-inflammatory and anti-platelet effects (16). Cyclooxygenase has 2 subtypes, COX-1 and COX-2, which promote the formation of thromboxane A2 and prostacyclin, respectively. Thromboxane A2 can activate platelets and constrict blood vessels, while prostacyclin plays the contrary roles. As a nonselective, irreversible inhibitor of cyclooxygenase, AA exerts stronger inhibition effects on COX-1. The drug DIP has vasodilative and antiplatelet effects. It can distend the uninvolved coronary arteries, reducing blood flow to the distal aneurysms and causing 'steal blood syndrome'. Using DIP in KD children complicated with CAL had risks in some degree, and DIP is usually used only in children with a salicylate contraindication, chicken pox infection, or influenza virus that requires suspension of AA (17). Meanwhile, DIP is a phosphodiesterase inhibitor with anti-platelet aggregation roles, and it inhibits platelet aggregation by increasing adenosine concentration, as well as inhibiting phosphodiesterase activity and reducing the formation of thromboxane A2 (TXA2). Warfarin can inhibit the liver to produce vitamin K-dependent clotting factors II, VII, $\mathrm{IX}$, and $\mathrm{X}$, as well as inhibit vitamin $\mathrm{K}$ cyclooxygenase, and vitamin $\mathrm{K}$ reductase as other clotting proteins. For KD children complicated with IV and V CAL, the INR should be kept between 1.5 and 2.5. Our study indicated that the anti-platelet effect of AA was significantly better than that of DIP, and the combination of AA with warfarin exerted synergetic effects in increasing AA inhibition ratio.

As a coumarin derivative, warfarin plays important roles in clinical anticoagulant therapy, especially for the 
treatment of KD children complicated with IV or $\mathrm{V}$ degree of CAL (1). The treatment window of warfarin is narrow, the treatment dose that achieves equal effect can be dozens of times different between individuals, and the risk of bleeding is high. The dose of warfarin is affected by genetic and non-genetic factors, and genetics are the main reason that contributed to the dose ranging. Studies have indicated that gene types with CYP2C9 and VKORC1 loci were the main factor affecting warfarin treatment dose (18). Warfarin is mainly metabolized by liver CYP2C9 into inactive 6- and 7-hydroxy compounds, and prevents the conversion of vitamin $\mathrm{K}$ from an oxidation-free form to an active hydroquinone form by vitamin $\mathrm{K}$ epoxide reductase complex subunit 1 (VKORC1), blocking the vitamin $\mathrm{K}$ dependent coagulation factors to exert anticoagulant roles $(19,20)$. Our study indicated that determination of warfarin dose according to the gene type could shorten the time of INR reaching standard in KD children, while dosage in the steady phase showed no difference between the routine treatment group and individual treatment group, which helps the doctors to evaluate and predict the side effects of warfarin, thus shorten the length of hospital stay of children. A limitation of our study was the relatively small sample size, and whether gene detection can guide clinical treatment requires further verification. A multicenter study involving large samples would be beneficial for normalizing the warfarin individual treatment to decrease bleeding risks.

Overall, the blood of children with KD is hypercoagulable in the acute phase, which further induces CAL. The TEG is of great significance for early recognition of the hypercoagulable state in KD children, and the $\mathrm{R}$ value can be used as a predictor of CAL in KD children. The KD children complicated with liver damage are more likely to experience coagulation dysfunction. Children with $\mathrm{KD}$ respond differently to various anti-coagulation drugs due to their genetic diversity. Evaluation of the effect of antithrombotic drugs can provide evidence for making an individualized treatment plan. Therefore, it is crucial to monitor the coagulation state and liver function of KD children after admission, and detect the genetic interaction of related drugs where necessary.

\section{Acknowledgments}

Funding: This study was supported by the Provincial Key Research and Development Program (18277708D).

\section{Footnote}

Reporting Checklist: The authors have completed the STROBE reporting checklist. Available at https://dx.doi. org/10.21037/tp-21-299

Data Sharing Statement: Available at https://dx.doi. org/10.21037/tp-21-299

Conflicts of Interest: All authors have completed the ICMJE uniform disclosure form (available at https://dx.doi. org/10.21037/tp-21-299). The authors declare no conflict of interest.

Ethical Statement: The authors are accountable for all aspects of this work in ensuring that questions related to the accuracy or integrity of any part of the work are appropriately investigated and resolved. The study was conducted in accordance with the Declaration of Helsinki (as revised in 2013). This study was approved by the Ethics Review Committee of Hebei Children's Hospital (2017005). All participants voluntarily participated in this study and their guardians provided written informed consent before treatment.

Open Access Statement: This is an Open Access article distributed in accordance with the Creative Commons Attribution-NonCommercial-NoDerivs 4.0 International License (CC BY-NC-ND 4.0), which permits the noncommercial replication and distribution of the article with the strict proviso that no changes or edits are made and the original work is properly cited (including links to both the formal publication through the relevant DOI and the license). See: https://creativecommons.org/ licenses/by-nc-nd/4.0/.

\section{References}

1. McCrindle BW, Rowley AH, Newburger JW, et al. Diagnosis, Treatment, and Long-Term Management of Kawasaki Disease: A Scientific Statement for Health Professionals From the American Heart Association. Circulation 2017;135:e927-99.

2. Levy DM, Silverman ED, Massicotte MP, et al. Longterm outcomes in patients with giant aneurysms secondary to Kawasaki disease. J Rheumatol 2005;32:928-34.

3. Saunders E, Ofili E. Epidemiology of atherothrombotic 
disease and the effectiveness and risks of antiplatelet therapy: race and ethnicity considerations. Cardiol Rev 2008;16:82-8.

4. de Graeff N, Groot N, Ozen S, et al. European consensusbased recommendations for the diagnosis and treatment of Kawasaki disease - the SHARE initiative. Rheumatology (Oxford) 2019;58:672-82.

5. Marchesi A, Rigante D, Cimaz R, et al. Revised recommendations of the Italian Society of Pediatrics about the general management of Kawasaki disease. Ital J Pediatr 2021;47:16.

6. Sakane T, Suzuki N, Nagafuchi H. Etiopathology of Behçet's disease: immunological aspects. Yonsei Med J 1997;38:350-8.

7. Zhou Y, Wang S, Zhao J, et al. Correlations of complication with coronary arterial lesion with VEGF, PLT, D-dimer and inflammatory factor in child patients with Kawasaki disease. Eur Rev Med Pharmacol Sci 2018;22:5121-6.

8. Chen X, Zhao ZW, Li L, et al. Hypercoagulation and elevation of blood triglycerides are characteristics of Kawasaki disease. Lipids Health Dis 2015;14:166.

9. Brogan P, Burns JC, Cornish J, et al. Lifetime cardiovascular management of patients with previous Kawasaki disease. Heart 2020;106:411-20.

10. Levi M, van der Poll T, Büller HR. Bidirectional relation between inflammation and coagulation. Circulation 2004;109:2698-704.

11. Hobson AR, Agarwala RA, Swallow RA, et al. Thrombelastography: current clinical applications and its potential role in interventional cardiology. Platelets 2006;17:509-18.

12. Besser MW. Post-operative of bleeding, haemolysis and coagulation in mechanical circulatory support patients.

Cite this article as: Hao J, Wang $\mathrm{H}$, Chen J, Li B, Zhang H, Zhang Y. Efficacy evaluation and mechanical study of shortand long-term antithrombotic therapy for Kawasaki disease. Transl Pediatr 2021;10(7):1883-1889. doi: 10.21037/tp-21-299
Ann Transl Med 2020;8:832.

13. Anjani G, Deglurkar R, Pilania RK, et al. Fulminant acute liver failure as an unusual presentation of Kawasaki disease. Scand J Rheumatol 2021;50:327-9.

14. Eladawy M, Dominguez SR, Anderson MS, et al. Abnormal liver panel in acute kawasaki disease. Pediatr Infect Dis J 2011;30:141-4.

15. Bader-Meunier B, Hadchouel M, Fabre M, et al. Intrahepatic bile duct damage in children with Kawasaki disease. J Pediatr 1992;120:750-2.

16. Agarwal S, Agrawal DK. Kawasaki disease: etiopathogenesis and novel treatment strategies. Expert Rev Clin Immunol 2017;13:247-58.

17. Research Committee of the Japanese Society of Pediatric Cardiology; Cardiac Surgery Committee for Development of Guidelines for Medical Treatment of Acute Kawasaki Disease. Guidelines for medical treatment of acute Kawasaki disease: report of the Research Committee of the Japanese Society of Pediatric Cardiology and Cardiac Surgery (2012 revised version). Pediatr Int. 2014;56(2):135-158.

18. Yang D, Kuang H, Zhou Y, et al. Height, VKORC1 1173, and CYP2C9 Genotypes Determine Warfarin Dose for Pediatric Patients with Kawasaki Disease in Southwest China. Pediatr Cardiol 2019;40:29-37.

19. Botton MR, Bandinelli E, Rohde LE, et al. Influence of genetic, biological and pharmacological factors on warfarin dose in a Southern Brazilian population of European ancestry. Br J Clin Pharmacol 2011;72:442-50.

20. Nguyen N, Anley P, Yu MY, et al. Genetic and clinical determinants influencing warfarin dosing in children with heart disease. Pediatr Cardiol 2013;34:984-90.

(English Language Editor: J. Jones) 